\title{
Efficient inhibition of foot-and-mouth disease virus replication in vitro by artificial microRNA targeting 3D polymerase
}

\author{
S. H. BASAGOUDANAVAR, H. B. RANJITHA, M. HOSAMANI, S. M. KOLANGATH, R. P. TAMIL \\ SELVAN, B. P. SREENIVASA, P. SARAVANAN, A. SANYAL, R. VENKATARAMANAN
}

ICAR-Indian Veterinary Research Institute, Hebbal, Bengaluru-560024, India

Received January 10, 2019; accepted April 30, 2019

\begin{abstract}
Summary. - Foot-and-mouth disease (FMD) is a devastating acute viral disease of livestock with cloven hooves. Among various therapeutic control measures, RNA interference (RNAi) is one of the methods being explored to inhibit FMD virus replication and spread. The RNAi is achieved by short hairpin RNAs or artificial microRNAs (amiRNAs). Utility of amiRNAs as antiviral, targeting conserved regions of the viral genome is gaining importance. However, delivery of miRNA in vivo is still a challenge. In this study, the efficacy of amiRNAs in preventing FMD virus replication in a permissive cell culture system was investigated, by generating stable cell lines expressing amiRNAs targeting three functional regions of the FMD virus (FMDV) genome (IRES, $3 \mathrm{~B} 3$ and $3 \mathrm{D}$ ). The results showed that amiRNA targeting 3D polymerase is relatively more efficient. However, expression of multiple microRNAs targeting the three regions did not exhibit additive effect. The data suggest that 3D specific miRNA is a potential valid strategy in developing novel antiviral measures against FMDV infection.
\end{abstract}

Keywords: artificial microRNA; foot-and-mouth disease virus; virus inhibition

\section{Introduction}

Foot-and-mouth disease virus (FMDV) is a pathogen of cloven hoofed animals, causing highly infectious and economically devastating disease. It is a major viral threat to livestock in many parts of the world, resulting in severe economic loss by way of impacting national and international trade of livestock products (Knight-Jones and Rushton, 2013). Seven serotypes (O, A, C, Asia-1, SAT-1, SAT-2 and SAT-3) of FMDV exist. In India, the disease is endemic with prevalence of serotypes $\mathrm{O}, \mathrm{A}$ and Asia-1. The virus belongs to the genus Aphthovirus (the family Picornaviridae) and has an icosahedral capsid containing positive sense single-stranded RNA. The viral genome is $8.5 \mathrm{~kb}$ long (Belsham, 1993), en-

E-mail: basagoudanavar.sh@icar.gov.in; phone: +91-80-23410729. Abbreviations: amiRNA $=$ artificial microRNA; FMD $=$ foot and-mouth disease; FMDV = FMD virus; $\mathrm{pi}=$ post infection; $\mathrm{UTR}=$ untranslated region coding a single long open reading frame (ORF) flanked by a long structured 5 '-untranslated region (5'-UTR) and a short 3'-UTR. The genome is enclosed within a non-enveloped icosahedral capsid made of 60 copies each of four different structural proteins - VP1, VP2, VP3 and VP4 (Curry et al., 1992; Fry et al., 2005). The virus encodes 10 non-structural proteins: L protease, $2 \mathrm{~A}, 2 \mathrm{~B}, 2 \mathrm{C}, 3 \mathrm{~A}, 3 \mathrm{~B}_{1-3}, 3 \mathrm{C}$ protease and 3D polymerase (Belsham, 1993; Clavijo et al., 2004). Virusspecific IRES element present within the 5 '-UTR is involved in translation of virus proteins (Lozano and Martinez-Salas, 2015). The $3 \mathrm{~B}_{3}$ is the important by the three similar but nonidentical copies of 3B protein (VPg) (Pacheco et al., 2003) that acts as peptide primer to synthesize viral RNA (Gao et al., 2016). The FMDV encoded RNA-dependent RNA polymerase (3D) (Robertson et al., 1983), is essential component of virus RNA replication. The sequences of these three regions - IRES, 3B3 and 3D are highly conserved among the different serotypes and subtypes of FMDV (Carrillo et al., 2005; George et al., 2001).

Foot-and-mouth disease (FMD) is a reportable animal disease, for which serotype-specific vaccination strategy is 
in practice to control the infection, as the serotypes fail to provide cross-protection (Jamal and Belsham, 2013). However, even after mass vaccination practice, sporadic disease outbreaks are still reported in endemic countries worldwide. Therefore, there is a need for effective antiviral(s) that can induce rapid protection in the event of FMD outbreaks in the endemic countries. Many antiviral molecules have been reported to inhibit FMDV, including 5-fluorouracil, 5-azacytidine, ribavirin etc. (Pariente et al., 2005). Until now, no efficacious antiviral against FMDV infection is available, although recent attempts using nucleoside analogues were encouraging, owing to concern of resistance to nucleoside analogues. Further, RNA interference using microRNAs (miRNAs) is being explored to inhibit replication of FMDV. The miRNAs are regulatory RNAs which modulate gene expression, via RNA induced silencing complex (RISC), by way of sequence-specific targeting of the regions of messenger RNAs or by causing translational repression of protein synthesis (He and Hannon, 2004). Interestingly, the expression of miRNAs is more effective and less toxic than regular siRNA/shRNA (McBride et al., 2008). The miRNA targeting integrin av receptor has been shown to give protection against FMDV in cell culture and transgenic suckling mice (Du et al., 2014). Further, antiviral effect of host miR-203a mimics against FMDV was reported previously (Gutkoska et al., 2017). We have recently shown that transient expression of host miRNAs appears to target FMD virus genomic RNA in cell culture (Basagoudanavar et al., 2018). Also, other studies have shown inhibitory effect of artificial miRNAs (amiRNAs) on FMDV, by targeting either the IRES sequence in the 5'-UTR, or 3'-UTR or the 3D polymerase (Chang et al., 2014; Du et al., 2011; Gismondi et al., 2014). The current study was undertaken to investigate the comparative potential of amiRNAs, targeted to different conserved FMDV sequences- IRES,3B3 and 3D regions, to inhibit FMDV replication in cell culture system.

\section{Materials and Methods}

Cell culture and virus. BHK-21 cells were maintained in Glasgow's modified Eagle's Medium (SAFC Biosciences, USA) supplemented with $10 \%$ fetal bovine serum. Serotype O IND-R2/75 FMD virus (Indian vaccine strain) was grown in BHK-21 cells.

Construction of plasmids expressing pre-miRNA. pre-miRNA sequences were designed using the guidelines of Block-it RNAi Web Designer tool (Invitrogen, USA), for 3B3, 3D and IRES regions from conserved sequences of FMDV (Table 1). The required sense and antisense oligos for desired amiRNAs were synthesized (Shrimpex Biotech Services, India) and annealed. The annealed pre-miRNAs were cloned into pcDNA ${ }^{\text {sn }} 6.2-\mathrm{GW} /$ $\mathrm{miR}$ vector, according to the manufacturer's protocol supplied with Block-iT Pol II miR RNAi Expression vector kit (Invitrogen, USA). The individual plasmids were named pcDNA6.2-miR-3B3, pcDNA6.2-miR-3D and pcDNA6.2-miR-IRES. To express the three miRNAs targeting IRES, 3B3, 3D in one primary transcript, the individual pre-miRNA expression cassettes were chained one after another according to the manufacturer's protocol supplied with Block-iT Pol II miR RNAi Expression vector kit (Invitrogen, USA). The plasmid expressing the multiple miRNAs was named pcDNA6.2-miR-IBD. As negative control, the pcDNA ${ }^{\mathrm{m}} 6.2-\mathrm{GW} /$ miR-neg control plasmid (Invitrogen, USA) which contains an insert that can form a hairpin structure that is processed into mature miRNA, but is predicted not to target any known vertebrate gene, was used. All the recombinant plasmids were screened by PCR. Plasmids were isolated from the putative positive colonies and restriction enzyme analysis was done using SnaBI and XhoI. The selected positive recombinant plasmids were further confirmed by nucleotide sequencing.

Generation of stable cell lines expressing miRNAs and analysis of virus inhibition. BHK-21 cells were transfected with recombinant plasmids expressing pre-miRNAs targeting FMDV-3B3, 3D, IRES, IBD and negative control miRNA. After $48 \mathrm{~h}$ gene delivery, selection was done using blasticidin $5 \mu \mathrm{g} / \mathrm{ml}$ for 5 days. Cells were split

Table 1. RNA hybrid between miRNA and target region in FMDV genome (serotype O IND-R2/75)

\begin{tabular}{|c|c|c|c|c|c|}
\hline \multirow{2}{*}{$\begin{array}{l}\text { amiRNA sequence }\left(5^{\prime} \text { to } 3^{\prime}\right) \\
\text { UUGAGUACCAGUAUCAGUGUC }\end{array}$} & \multirow{2}{*}{$\frac{\text { Target region }}{\text { IRES }}$} & \multicolumn{4}{|c|}{ miRNA (bottom) hybrid with target sequence (top) } \\
\hline & & $5^{\prime}$ & $\mathrm{U}$ & A & $3^{\prime}$ \\
\hline & & \multicolumn{4}{|c|}{$\begin{array}{l}\text { GACACUGAUACUGGUACUCAA } \\
\text { CUGUGACUAUGACCAUGAGUU }\end{array}$} \\
\hline & & $3^{\prime}$ & & & $5^{\prime}$ \\
\hline \multirow[t]{4}{*}{ UUCUUCACCGGUCCCUCGUAA } & 3B3 & $5^{\prime}$ & $\mathrm{C}$ & G & $3^{\prime}$ \\
\hline & & \multirow{2}{*}{\multicolumn{4}{|c|}{$\begin{array}{l}\text { UUACGAGGGGCCGGUGAAGAA } \\
\text { AAUGCUCCCUGGCCACUUCUU }\end{array}$}} \\
\hline & & & & & \\
\hline & & $3^{\prime}$ & & & $5^{\prime}$ \\
\hline \multirow[t]{4}{*}{ UUGAUUGCCUCGUAGAUGCUC } & $3 \mathrm{D}$ & $5^{\prime}$ & $\mathrm{U}$ & G & $3^{\prime}$ \\
\hline & & \multirow{2}{*}{\multicolumn{4}{|c|}{$\begin{array}{l}\text { GAGCAUCUACGAGGCAAUCAA } \\
\text { CUCGUAGAUGCUCCGUUAGUU }\end{array}$}} \\
\hline & & & & & \\
\hline & & $3^{\prime}$ & G & & $5^{\prime}$ \\
\hline
\end{tabular}

*Generated using online tool- https://bibiserv2.cebitec.uni-bielefeld.de/rnahybrid (Rehmsmeier et al., 2004). 
before selection with optimized cell density $\left(5 \times 10^{4}\right.$ cells/well $)$ and were analysed after 10 passages by PCR using pcDNA-SeqF (which binds in CMV promoter) and M13 reverse primer for the presence of the pre-miRNA coding construct in the cell line. The cells were maintained under selection pressure at all times to generate stable BHK-21 cell line expressing miRNA targeting IRES, 3B3 and 3D regions or the three miRNAs together.

To evaluate inhibitory effect of the miRNAs targeting IRES, $3 \mathrm{~B} 3$ and 3D on FMDV replication, stably expressing BHK-21 cell lines were infected with FMDV, at multiplicity of infection (MOI) of 0.001. Cell supernatant was harvested at 6, 12 and $24 \mathrm{~h}$ post infection (pi). Progeny virus in the culture supernatant was analysed by titration using TCID $_{50}$ assay as described earlier (Reed and Muench, 1938). Percent reduction (P) in titre was calculated using the formula, $\mathrm{P}=\left(1-10^{-\mathrm{L}}\right) \times 100$ where $\mathrm{L}$ is the $\mathrm{Log}$ reduction (http://microchemlab.com/log reduction and percent reduction calculations).

Western blot analysis. To analyze the protein levels of 3D in BHK-21 cell lines expressing 3D specific miRNAs, the cells were seeded in a 6-well plate at a density of $2 \times 10^{5} \mathrm{cells} /$ well. They were infected at an MOI of 0.001 with FMDV. The cells were harvested at 12, 24 and 30 hpi and cell lysate was prepared using RIPA lysis buffer. Equal amounts of the proteins were separated on $10 \%$ SDS-PAGE and transferred onto PVDF membranes (Millipore Corporation, USA) by semidry blotting. The membrane was blocked in phosphate-buffered saline with $0.1 \%$ Tween 20 (PBST) containing 3\% skimmed milk for $2 \mathrm{~h}$ and then incubated with primary antibody (anti-3D monoclonal antibody 6B8D11, 1:100; rabbit anti-actin, 1:500) at $4^{\circ} \mathrm{C}$ overnight. Following washing with PBST, the membrane was incubated with the secondary antibody (HRP-conjugated goat anti-mouse/rabbit IgG; DAKO, Denmark) at room temperature for $1 \mathrm{~h}$. The membrane was then washed with PBST, and developed using Supersignal west pico chemilumines-

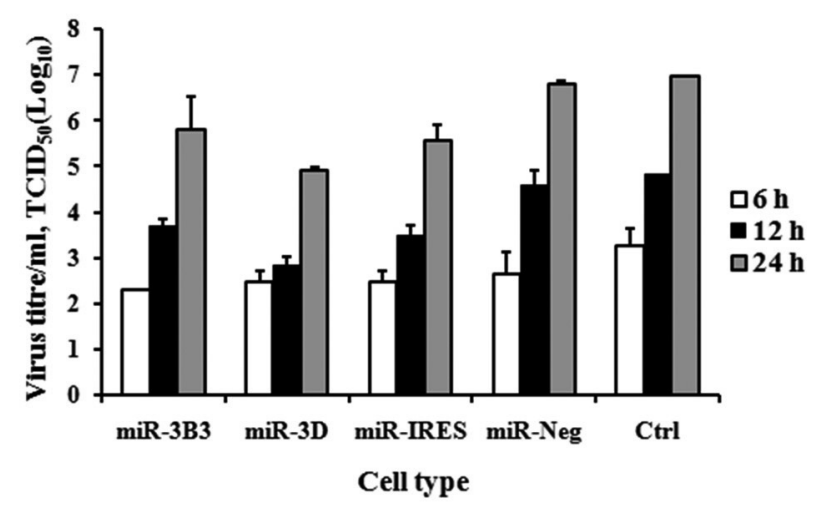

Fig. 1

Inhibition of FMD virus yields in BHK-21 with stable expression of pre-miRNAs targeting 3B3, 3D or IRES

Bar diagram represents mean $\pm \mathrm{SD}$ of $\log _{10}$ value of progeny virus titre at indicated time points post infection with MOI of 0.001 , analysed by titration using TCID $_{50}$ assay. cent substrate (Thermo Fisher Scientific, USA), and imaged using chemiluminescent Imager (UVITEC, UK).

Statistical analysis. The significance of differences in means was determined by Student's $t$ test and $p<0.05$ was considered statistically significant.

\section{Results and Discussion}

To investigate the inhibitory effect of the miRNAs targeting IRES, 3B3 and 3D, on FMDV replication, progeny virus in the culture supernatant harvested at 6, 12 and $24 \mathrm{~h}$ pi, from stable cell line, was analysed by titration using TCID $_{50}$ assay. At $6 \mathrm{~h} \mathrm{pi}$, the progeny virus titre was lower in the miRNA expressing cells compared to negative control miRNA, though not statistically significant $(p>0.05)$. However, cells with miRNAs targeting IRES, 3B3 and 3D showed significant reduction $(p<0.05)$ in titre compared to negative control miRNA, at $12 \mathrm{~h}$ pi. At $24 \mathrm{~h}$ pi, only IRES and 3D specific miRNA showed significantly $(p<0.05)$ reduced progeny virus titre compared to negative control miRNA. The miRNA targeting 3D sequence had $\sim 2 \log _{10}$ reduction in virus titre (99\% inhibition of virus titre) at $24 \mathrm{~h}$ pi, compared to negative control miRNA. The miRNA targeting IRES and 3B3 sequences respectively showed 1.25 (94\% inhibition of virus titre) and $1 \log _{10}$ reduction in virus titre (90\% inhibition of virus titre), respectively (Fig. 1). Thus, it was evident that miRNA mediated targeting of $3 \mathrm{D}$ polymerase is most effective in inhibiting FMDV in cell culture. Previously, it was reported that cell lines constitutively expressing amiRNAs targeting FMDV 3D coding region or 3'-UTR showed variable silencing effects, possibly due to accessibility of target sites (Gismondi et al., 2014). However, our data clearly show

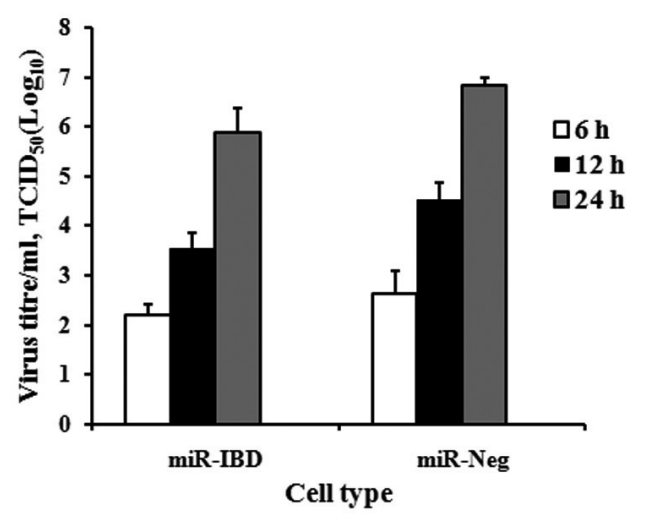

Fig. 2

Inhibition of FMD virus yields in BHK-21 with stable expression of multiple pre-miRNAs targeting IRES, 3B3 and 3D together Bar diagram represents mean $\pm \mathrm{SD}$ of $\log _{10}$ value of progeny virus titre at indicated time points post infection with MOI of 0.001 , analysed by titration using TCID $_{50}$ assay. 


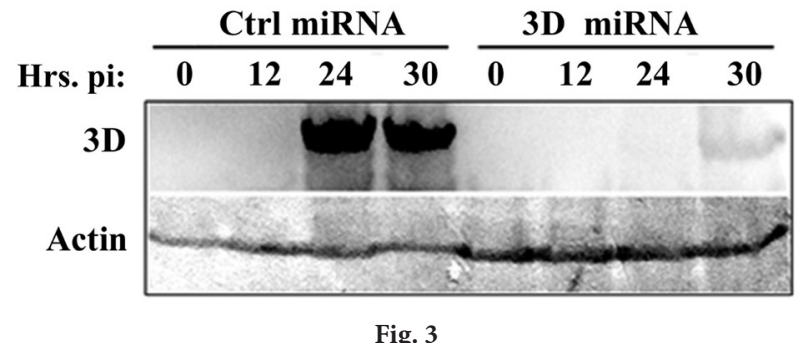

Fig. 3

Inhibition of FMD virus 3D protein expression in BHK-21 with stable expression of $3 \mathrm{D}$ specific pre-miRNA

Western blot analysis of 3D protein showing reduced virus multiplication in BHK-21 cells expressing pre-miRNA targeting 3D polymerase, compared to cell line expressing negative control pre-miRNA. Actin was used as loading control.

reproducible effectiveness of amiRNAs targeting FMDV 3D region, in hindering virus replication.

It was earlier reported that multiple miRNA targeting is more effective. IRES-specific dual-miRNA, expressing two miRNA hairpin structures was able to effectively inhibit the replication of FMDV (Chang et al., 2014). Therefore, we attempted to establish a cell line that expressed amiRNA targeting 3B3, 3D and IRES regions together. Upon analysing the progeny virus in the culture supernatant harvested at 6 , 12 and $24 \mathrm{~h}$ pi, there was significant $(p<0.05)$ titre reduction at 12 and $24 \mathrm{~h}$ pi (Fig. 2). However, there was no cumulative effect as was expected from the effect of 3 amiRNAs. Infact, the virus inhibition was less than that observed by $3 \mathrm{D}$ or IRES specific amiRNAs independently. This could be due to promoter competition (Conte et al., 2002) for the expression of the three miRNAs by the same construct.

Western blot for 3D protein from the cell lysate of BHK-21 expressing pre-miRNA targeting 3D polymerase, also showed significantly reduced protein level suggesting restricted virus multiplication, compared to cell line expressing pre-miRNA negative control (Fig. 3). The reduction in protein level is due to specific targeting of 3D by the miRNA. Interestingly, while there was drastically reduced protein detection by western blot analysis, the reduction in virus titre was only $\log _{10} 2$. This might be due to the fact that even a small amount of active 3D polymerase could be enough to drive acute replication of FMDV.

The current FMD control program in several affected countries focuses on regular vaccination with inactivated virus to maintain herd immunity. However, sporadic outbreaks of FMD are still reported. Therefore, there is a need for development of emergency antiviral strategies that can induce rapid protection to prevent disease spread. Previous study in cattle (Charleston et al., 2011) suggests that there is a narrow window of opportunity to detect and segregate affected animals before they pass on the infection to healthy ones. The possibility of FMD virus transmission could be reduced if early control measures are executed. In this context, miRNAs which target virus genome are a new hope in restricting the virus at the early stages of its life cycle in the cell, with ultimate potential application in minimizing virus spread during outbreak.

In conclusion, this study compared the efficacy of amiRNA targeting three viral sequences (3D, 3B and IRES) in inhibition of FMDV replication. Our results demonstrated that amiRNA targeting 3D region showed highly efficient inhibition as compared to other conserved targets in the virus sequence. Further studies are warranted for the in vivo application of this strategy for therapeutic exploitation, to reduce FMDV transmission in endemic regions.

Acknowledgment. We acknowledge the Director, ICAR-Indian Veterinary Research Institute (IVRI) Izatnagar, for facilitating this work.

\section{References}

Basagoudanavar SH, Hosamani M, Tamil Selvan RP, Sreenivasa BP, Sanyal A, Venkataramanan R (2018): Host serum microRNA profiling during the early stage of foot-and-mouth disease virus infection. Arch. Virol. 163, 2055-2063. https://doi.org/10.1007/s00705-018-3824-8

Belsham GJ (1993): Distinctive features of foot-and-mouth disease virus, a member of the picornavirus family; aspects of virus protein synthesis, protein processing and structure. Prog. Biophys. Mol. Biol. 60, 241-260. https://doi. org/10.1016/0079-6107(93)90016-D

Carrillo C, Tulman ER, Delhon G, Lu Z, Carreno A, Vagnozzi A, Kutish GF, Rock DL (2005): Comparative genomics of foot-and-mouth disease virus. J. Virol. 79, 6487-6504. https://doi.org/10.1128/JVI.79.10.6487-6504.2005

Chang Y, Dou Y, Bao H, Luo X, Liu X, Mu K, Liu Z, Liu X, Cai X (2014): Multiple microRNAs targeted to internal ribosome entry site against foot-and-mouth disease virus infection in vitro and in vivo. Virol. J. 11, 1. https://doi. org/10.1186/1743-422X-11-1

Charleston B, Bankowski BM, Gubbins S, Chase-Topping ME, Schley D, Howey R, Barnett PV, Gibson D, Juleff ND, Woolhouse ME (2011): Relationship between clinical signs and transmission of an infectious disease and the implications for control. Science 332, 726-729. https:// doi.org/10.1126/science.1199884

Clavijo A, Wright P, Kitching P (2004): Developments in diagnostic techniques for differentiating infection from vaccination in foot-and-mouth disease. Vet. J. 167, 9-22. https://doi. org/10.1016/S1090-0233(03)00087-X

Conte C, Dastugue B, Vaury C (2002): Promoter competition as a mechanism of transcriptional interference mediated by retrotransposons. EMBO J. 21, 3908-3916. https://doi. org/10.1093/emboj/cdf367

Curry S, Abu-Ghazaleh R, Blakemore W, Fry E, Jackson T, King A, Lea S, Logan D, Newman J, Stuart D (1992): Crystal- 
lization and preliminary X-ray analysis of three serotypes of foot-and-mouth disease virus. J. Mol. Biol. 228, 12631268. https://doi.org/10.1016/0022-2836(92)90332-E

Du J, Gao S, Luo J, Zhang G, Cong G, Shao J, Lin T, Cai X, Chang $\mathrm{H}$ (2011): Effective inhibition of foot-and-mouth disease virus (FMDV) replication in vitro by vector-delivered microRNAs targeting the 3D gene. Virol. J. 8, 292. https:// doi.org/10.1186/1743-422X-8-292

Du J, Guo X, Gao S, Luo J, Gong X, Hao C, Yang B, Lin T, Shao J, Cong G, Chang, H (2014): Induction of protection against foot-and-mouth disease virus in cell culture and transgenic suckling mice by miRNA targeting integrin alphav receptor. J. Biotechnol. 187, 154-161. https://doi. org/10.1016/j.jbiotec.2014.07.001

Fry EE, Stuart DI, Rowlands DJ (2005): The structure of foot-andmouth disease virus. Curr. Top. Microbiol. Immunol. 288, 71-101. https://doi.org/10.1007/3-540-27109-0 4

Gao Y, Sun SQ, Guo HC (2016): Biological function of Foot-andmouth disease virus non-structural proteins and noncoding elements. Virol. J. 13, 107. https://doi.org/10.1186/ $\underline{\text { s12985-016-0561-Z }}$

George M, Venkataramanan R, Pattnaik B, Sanyal A, Gurumurthy CB, Hemadri D, Tosh C (2001): Sequence analysis of the RNA polymerase gene of foot-and-mouth disease virus serotype Asia1. Virus Genes 22, 21-26. https://doi. org/10.1023/A:1011153904910

Gismondi MI, Ortiz XP, Curra AP, Asurmendi S, Taboga O (2014): Artificial microRNAs as antiviral strategy to FMDV: structural implications of target selection. J. Virol. Methods 199, 1-10. https://doi.org/10.1016/j. jviromet.2013.12.016

Gutkoska J, LaRocco M, Ramirez-Medina E, de Los Santos T, Lawrence P (2017): Host microRNA-203a is antagonistic to the progression of foot-and-mouth disease virus infection. Virology 504, 52-62. https://doi.org/10.1016/j. virol.2017.01.019

He L, Hannon GJ (2004): MicroRNAs: small RNAs with a big role in gene regulation. Nat. Rev. Genet. 5, 522-531. https:// doi.org/10.1038/nrg1379
Jamal SM, Belsham GJ (2013): Foot-and-mouth disease: past, present and future. Vet Res 44, 116. https://doi.org/10.1186/ 1297-9716-44-116

Knight-Jones TJ, Rushton J (2013): The economic impacts of foot and mouth disease - what are they, how big are they and where do they occur? Prev. Vet. Med. 112, 161-173. https://doi.org/10.1016/j.prevetmed.2013.07.013

Lozano G, Martinez-Salas E (2015): Structural insights into viral IRES-dependent translation mechanisms. Curr. Opin. Virol. 12, 113-120. https://doi.org/10.1016/j. coviro.2015.04.008

McBride JL, Boudreau RL, Harper SQ, Staber PD, Monteys AM, Martins I, Gilmore BL, Burstein H, Peluso RW, Polisky B, Carter BJ, Davidson BL (2008): Artificial miRNAs mitigate shRNA-mediated toxicity in the brain: implications for the therapeutic development of RNAi. Proc. Natl. Acad. Sci. USA 105, 5868-5873. https://doi.org/10.1073/ pnas. 0801775105

Pacheco JM, Henry TM, O'Donnell VK, Gregory JB, Mason PW (2003): Role of nonstructural proteins $3 \mathrm{~A}$ and $3 \mathrm{~B}$ in host range and pathogenicity of foot-and-mouth disease virus. J. Virol. 77, 13017-13027. https://doi.org/10.1128/ JVI.77.24.13017-13027.2003

Pariente N, Sierra S, Airaksinen A (2005): Action of mutagenic agents and antiviral inhibitors on foot-and-mouth disease virus. Virus Res. 107, 183-193. https://doi.org/10.1016/j. virusres.2004.11.008

Reed LJ, Muench H (1938): A simple method of estimating fifty percent endpoints. American J. Hyg. 27, 493-497. https:// doi.org/10.1093/oxfordjournals.aje.a118408

Rehmsmeier M, Steffen P, Hochsmann M, Giegerich R (2004): Fast and effective prediction of microRNA/target duplexes. RNA 10, 1507-1517. https://doi.org/10.1261/rna.5248604

Robertson BH, Morgan DO, Moore DM, Grubman MJ, Card J, Fischer T, Weddell G, Dowbenko D, Yansura D (1983): Identification of amino acid and nucleotide sequence of the foot-and-mouth disease virus RNA polymerase. Virology 126, 614-623. https://doi.org/10.1016/S0042$\underline{6822(83) 80017-8}$ 